OPEN ACCESS

Edited by:

Martin G. Klotz,

Queens College, City University

of New York, USA

Reviewed by:

James Hemp,

California Institute of Technology, USA

Eric Boyd,

Montana State University, USA

${ }^{*}$ Correspondence:

Dmitry A. Ravcheev

dmitry.ravcheev@uni.lu

Mikhail S. Gelfand

gelfand@iitp.ru

${ }^{\dagger}$ These authors have contributed equally to this work.

Specialty section:

This article was submitted to Evolutionary and Genomic

Microbiology,

a section of the journal

Frontiers in Microbiology

Received: 09 March 2016 Accepted: 15 August 2016

Published: 26 August 2016

Citation:

Tsoy OV, Ravcheev DA, Čuklina J and Gelfand MS (2016) Nitrogen

Fixation and Molecular Oxygen:

Comparative Genomic

Reconstruction of Transcription

Regulation in Alphaproteobacteria.

Front. Microbiol. 7:1343.

doi: 10.3389/fmicb.2016.01343

\section{Nitrogen Fixation and Molecular Oxygen: Comparative Genomic Reconstruction of Transcription Regulation in Alphaproteobacteria}

\author{
Olga V. Tsoy ${ }^{1 \dagger}$, Dmitry A. Ravcheev2*t, Jelena Čuklina1,3 and Mikhail S. Gelfand ${ }^{1,4,5,6 *}$ \\ ${ }^{1}$ Research and Training Center on Bioinformatics, A.A. Kharkevich Institute for Information Transmission Problems, Russian \\ Academy of Sciences, Moscow, Russia, ${ }^{2}$ Luxembourg Centre for Systems Biomedicine, University of Luxembourg, \\ Esch-sur-Alzette, Luxembourg, ${ }^{3}$ Moscow Institute of Physics and Technology, Dolgoprudny, Russia, ${ }^{4}$ Faculty of \\ Bioengineering and Bioinformatics, Moscow State University, Moscow, Russia, ${ }^{5}$ Skolkovo Institute of Science and \\ Technology, Skolkovo, Russia, ${ }^{6}$ Faculty of Computer Science, Higher School of Economics, Moscow, Russia
}

Biological nitrogen fixation plays a crucial role in the nitrogen cycle. An ability to fix atmospheric nitrogen, reducing it to ammonium, was described for multiple species of Bacteria and Archaea. The transcriptional regulatory network for nitrogen fixation was extensively studied in several representatives of the class Alphaproteobacteria. This regulatory network includes the activator of nitrogen fixation NifA, working in tandem with the alternative sigma-factor RpoN as well as oxygen-responsive regulatory systems, one-component regulators FnrN/FixK and two-component system FixLJ. Here we used a comparative genomics approach for in silico study of the transcriptional regulatory network in 50 genomes of Alphaproteobacteria. We extended the known regulons and proposed the scenario for the evolution of the nitrogen fixation transcriptional network. The reconstructed network substantially expands the existing knowledge of transcriptional regulation in nitrogen-fixing microorganisms and can be used for genetic experiments, metabolic reconstruction, and evolutionary analysis.

Keywords: bacteria, transcription factors, nitrogen fixation, comparative genomics, regulons

\section{INTRODUCTION}

Nitrogen is indispensable for all living species. The Earth atmosphere mainly consists of nitrogen, but in the form of dinitrogen $\left(\mathrm{N}_{2}\right)$ which is not available for living organisms. Only some Bacteria and Archaea can convert $\mathrm{N}_{2}$ into ammonium $\left(\mathrm{NH}_{3}\right)$ to further synthesize organic compounds. This process is known as biological nitrogen fixation. Biological nitrogen fixation plays a crucial role in the nitrogen cycle as it returns the element from the geosphere and atmosphere to living species (reviewed in Dixon and Kahn, 2004).

Species capable of nitrogen fixation belong to Bacteria and Archaea, as no examples of nitrogen fixation in Eukarya are known. Within Archaea, nitrogen fixation has been observed in some methanogens (Methanobacteriales, Methanococcales, and Methanosarcinales). 
In Bacteria, nitrogen fixation is much more widely distributed and has been characterized in phyla Actinobacteria, Bacteroidetes, Cyanobacteria, Chlorobi, Chloroflexi, Firmicutes, and Proteobacteria (Raymond et al., 2004; Dos Santos et al., 2012; Boyd and Peters, 2013).

Nitrogen fixation is often the limiting factor for crop and natural ecosystem productivity making this process important for agriculture (Dixon and Kahn, 2004). It is carried out that a large number of nitrogen fixing bacteria in soils and in symbioses with plants are Alphaproteobacteria.

The main enzyme catalyzing reduction of $\mathrm{N}_{2}$ to ammonium is the nitrogenase. All known nitrogenases require a FeS-cluster and other metal-dependent cofactors for electron transduction. The best studied and the most common is the molybdenumdependent nitrogenase which is encoded by the nifHDK genes (Boyd and Peters, 2013). Alternative nitrogenases are the vanadium- and iron-dependent nitrogenases encoded by the $v n f H D G K$ and anfHDGK genes, respectively (Rehder, 2000; Seefeldt et al., 2009; Hartmann and Barnum, 2010). Since nitrogenases are irreversibly inhibited by dioxygen $\left(\mathrm{O}_{2}\right)$ (Gallon, 1981; Boyd and Peters, 2013), bacteria developed various strategies to avoid the latter, such as elimination of $\mathrm{O}_{2}$ through enzyme-catalyzed reactions, symbiotic nodules, compartmentalization etc (Oelze, 2000; Boyd et al., 2015).

In spite of the importance of nitrogen fixation, the regulation of this process has been well described in only a few bacterial species. Diazotrophs have developed multiple strategies in regulation of nitrogen fixation. Some regulate nitrogen fixation post-translationally (Huergo et al., 2012). For example, in Rhodobacter capsulatus nitrogenase can be by reversibly inactivated by the DraT protein (Masepohl et al., 2002). Proteins NifL and $\mathrm{NifI}_{1} \mathrm{I}_{2}$, involved in post-translational regulation in Klebsiella pneumoniae (Dixon and Kahn, 2004) and Heliobacterium chlorum (Enkh-Amgalan et al., 2006) respectively, are absent in Alphaproteobacteria (Boyd et al., 2015). PII proteins, critical for Herbaspirillum seropedicae, have been shown to be non-essential for nitrogen fixation in some Alphaproteobacteria (Arcondeguy et al., 1997; Yurgel et al., 2010).

From early studies on nitrogen fixation in rhizobia it is known, that nitrogen fixation cannot occur in nifA, fixJ, and fixK mutants. All these genes encode transcription factors (Hennecke, 1990). In Proteobacteria, the nitrogenase genes are invariably activated by NifA, while FixJ and FixK were shown to control nitrogen fixation in response to microaerobic conditions in certain Rhizobiales (Fischer, 1994). The regulation by NifA, FixJ, and FixK has been well described in only a few model species. Whether functions regulated by these factors span beyond regulation of nitrogen fixation, and what are other possible members of this regulatory network, what are the relationships between these regulators, and to what extent these networks are conserved in different species is not clear.

In most nitrogen-fixing bacteria NifA is the master regulator of nitrogen fixation. It works in association with the RNApolymerase sigma factor RpoN (sigma54; Sullivan et al., 2002; Sciotti et al., 2003). RpoN recognizes a $-24 /-12$ promoter sequence with the consensus TGGCACG-N 4 -TTGCW and binds the NifA protein which in turn recognizes its own binding site with the consensus sequence TGT-N 10 -ACA (Barrios et al., 1999). As mentioned above, nitrogen fixation is very sensitive to $\mathrm{O}_{2}$ concentration, and NifA has an ability to sense it through conserved cysteine residues. While NifA effects nitrogen fixation itself, FixLJ and FnrN/FixK are responsible for the cell adaptation to microaerobic conditions (Sciotti et al., 2003).

FixJ is a DNA-binding response regulator working in tandem with sensor kinase FixL, constituting a two-component regulatory system FixLJ (Reyrat et al., 1993; Torres et al., 2011). FixL directly senses $\mathrm{O}_{2}$, which binds the haem group in the sensor domain and inactivates its kinase activity (Rey and Harwood, 2010; Sousa et al., 2015). FixJ is one of the major regulators of nitrogen fixation, but the only known conserved member of the FixJ regulon is fixK (Nellen-Anthamatten et al., 1998). Several attempts have been made to determine its binding motif, leading to varying results. Sequence analysis of the fixK promoter region in Sinorhizobium. meliloti, Azorhizobium caulinodans and Bradyrhizobium japonicum yielded a common CSNAATWT motif at position -33 and an additional TAAG element around position -64 (Sciotti et al., 2003). This observation was confirmed by DNase protection which showed that S. meliloti FixJ binds to the fixK promoter in two different regions $(-69$, $-44)$ and $(-57$ and -31$)$ relative to the transcription start site (Galinier et al., 1994). The application of the SELEX technique predicted two classes of FixJ-binding sites with the consensus motifs GTAGTTTCCC and GTAMGTAG (Ferrieres and Kahn, 2002). The most recent studies analyzed FixJ-binding sites in $S$. meliloti using a gel shift assay and the NMR structure of the truncated C-terminal FixJ DNA-binding domain. They determined the TAAGTAATTTCCCTTA sequence in the upstream region of the fixK gene as the FixJ-binding site (Kurashima-Ito et al., 2005). Therefore, the lack of a common consensus complicates identification of new FixJ targets.

Some Rhizobiales, in particular, R. etli and R. leguminosarum, lack FixJ, and thus use an alternative regulatory cascade comprised of a hybrid histidine kinase hFixL and a response regulator FxkR, analogous to FixJ and FixKf. A FxkR-binding site with the consensus GTTACA-N 4 -GTTACA was identified upstream of the fixKf gene by MEME and confirmed by the gel shift assay (Zamorano-Sanchez et al., 2012).

Other regulators of nitrogen fixation, FixK and FnrN, belong to the Crp-Fnr superfamily of transcription factors (Matsui et al., 2013; Rodgers et al., 2013). The main difference between these proteins is the ability of FnrN to directly sense oxygen with the iron-sulfur cluster. The cluster is formed by the conserved $\mathrm{N}$-terminal cysteine-rich domain and an additional cysteine in the middle of the polypeptide chain (Korner et al., 2003). FixK and FnrN have been studied extensively in Alphaproteobacteria and shown to control expression of oxidases, hydrogen uptake, nitrogen metabolism, haem biosynthesis, and nitrogen-fixation transcription factors (Supplementary Table S1 in the Supplementary Materials). The existence of two closely related regulators, FnrN and FixK, is the main obstacle for the genomic analysis of their regulatory interactions, as both transcription factors recognize very similar binding sites with the consensus motif TTGANCNNGATCAANG (Cebolla 
and Palomares, 1994; Mesa et al., 2005, 2008; Bonnet et al., 2013).

Hence, known regulatory networks for nitrogen fixation in Alphaproteobacteria are rather complex, may vary between species, and seem highly redundant due to the presence of multiple transcription factors with very similar functions. The experimental data about transcriptional regulation of nitrogen fixation are difficult to obtain and thus are generated at a slow rate. On the other hand, the growing number of complete bacterial genomes and the development of comparative genomics techniques allow us to analyze transcriptional regulatory systems, addressing relevant biological questions. Here we report the results of manual comparative analysis of the regulatory network for nitrogen fixation in 50 complete genomes belonging to five orders of Alphaproteobacteria. We predict new members of the nitrogen fixation regulatory network and reconstruct different types of regulatory cascades found in Alphaproteobacteria. Based on these predictions, we propose a model of the gradual growth of complexity for this network and reconstruct possible evolutionary events that have formed the network. We also have constructed taxon-specific profiles for each of the analyzed regulators. The obtained dataset including the information about the analyzed transcription regulators, their binding sites and motifs, and regulated genes is deposited in the RegPrecise database (Novichkov et al., 2013).

\section{MATERIALS AND METHODS}

\section{Comparative Genomics Approach for the Reconstruction of Regulons}

For the regulon reconstruction, we used a comparative genomics approach (Rodionov, 2007) implemented in the RegPredict Web server (Novichkov et al., 2010). The workflow includes inference of transcription regulator binding sites, construction of positional weight matrices (profiles) for the binding-site motifs, and further search for additional regulon members based on predicted binding sites in gene promoter regions. To take into account possible lineage-specific changes in binding site motifs, we constructed individual profiles for genomes from each considered order of Alphaproteobacteria.

To construct the profiles, the following steps were implemented. An initial profile was constructed based on known binding sites of a given regulator or on sequences of binding sites predicted by phylogenetic footprinting, an approach based on the analysis of conserved islands in multiple alignments of DNA fragments (Shelton et al., 1997; McCue et al., 2001). This profile was used for site prediction in genomes from a given order. Two types of predicted sites were used to update the taxon-specific profile: (1) sites predicted upstream of orthologs of genes known to be regulated in at least one genome and (2) sites upstream of operons with conserved predicted regulation, i.e., sites that were found upstream of orthologous genes in at least than half of genomes from the analyzed order (the consistency check approach; Ravcheev et al., 2007; Rodionov, 2007). Operons were defined as groups of genes satisfying the following criteria: same direction of transcription, intergenic distance not exceeding $200 \mathrm{bp}$, absence of internal binding sites, and conservation of the locus structure in a number of related genomes. The obtained order-specific profile was further used for the analysis of regulons in this order.

The initial profiles for the NifA and FnrN/FixK-like proteins were constructed based on sequences of known binding sites extracted from literature data. For the FixJ and FxkR proteins, the initial profiles were constructed using phylogenetic footprinting. The constructed profiles were further used to search for additional regulon members using the Run Profile tool in RegPredict. The lowest score observed in the training set of known and/or initially predicted binding sites was used as the threshold for the genome-wide site search. To eliminate false positives, the consistency check approach or functional relevance of candidate target operons were used.

\section{Tools and Databases}

Fifty studied genomes were downloaded from the MicrobesOnline database (Dehal et al., 2010). The initial search for FnrN and FixK homologs was done using BLASTP (Altschul et al., 1997) with known FnrN/FixK proteins (cutoff: $e$-value $=\mathrm{e}-30$, identity $=40 \%)$. The domain structure of defined homologs was predicted with the Pfam database (Finn et al., 2014) and used as an additional criterion: a protein should the have $\mathrm{N}$-terminal cyclic nucleotide-binding domain (PF00027) and C-terminal Crp-like helix-turn-helix domain (PF13545). Gene orthology was determined by the bidirectional best hit criterion implemented in the GenomeExplorer software (Mironov et al., 2000) and validated by phylogenetic trees from the MicrobesOnline database. Genes were considered as orthologs if they: (1) formed a monophyletic branch in the phylogenetic tree; and (2) demonstrated conserved chromosomal gene context. Functional gene annotations were extracted from the literature and uploaded from the SEED (Disz et al., 2010), UniProt (Magrane and Consortium, 2011), MicrobesOnline, and KEGG (Kanehisa et al., 2014) databases.

Multiple alignments for amino acid and nucleotide sequences were constructed using a web version ${ }^{1}$ of the MUSCLE tool (Edgar, 2004) with default parameters. Phylogenetic trees were constructed by the maximum-likelihood method (Felsenstein, 1996) and LG model for amino acid substitution (Le and Gascuel, 2008) implemented in -3.0 (Guindon et al., 2010) with default parameters, and visualized with the Dendroscope (version 3.2.10, built 19) program (Huson et al., 2007). Sequence logos for DNA motifs were drawn with WebLogo (Crooks et al., 2004).

\section{Data Availability}

All predicted regulons including transcription regulators, their binding sites, their regulated genes and operons, and functional gene assignments are deposited in the RegPrecise database (Novichkov et al., 2013) and are freely available at http:// regprecise.lbl.gov/RegPrecise (Supplementary Table S2 in the Supplementary Materials).

\footnotetext{
${ }^{1}$ http://www.ebi.ac.uk/Tools/msa/muscle
} 


\section{RESULTS}

\section{Distribution of Genes Encoding the Nitrogenase and the Transcription Regulators}

To describe the transcriptional regulation for nitrogen fixation systematically among Alphaprpteobacteria, we started with the analysis of the distribution of genes encoding the nitrogenase and the regulatory proteins in 50 genomes of Alphaproteobacteria (Supplementary Table S3 in the Supplementary Materials).

\section{nifHDK}

The nitrogenase-encoding genes nifHDK were found in most Rhizobiales and Rhodospirillales, and were not found in Caulobacteriales. The nifHDK genes were also detected in one Rhodobacterales genome, Rhodobacter sphaeroides 2.4.1, and one Sphingomonadales genome, Zymomonas mobilis ZM4.

\section{NifA}

The orthologs of the transcription regulator NifA were found in 10 Rhizobiales, 6 Rhodospirillales, 1 Sphingomonadales, and 1 Rhodobacterales genomes. No NifA orthologs were found in Caulobacteriales. The NifA genes are always co-localized in genome with the nifHDK genes, and in 10 studied genomes the nifA genes are co-localized with the former (Supplementary Table S3 in the Supplementary Materials).

\section{FixLJ}

Orthologs of the FixLJ two-component system were identified in all four analyzed Caulobacteriales and in 15 of 20 analyzed Rhizobiales. Previously, an alternative cascade called hFixL-FxkRFixKf has been identified in several Rhizobiales (ZamoranoSanchez et al., 2012). Among genomes studied here, the orthologs of these transcription factors were found in $R$. etli, R. leguminosarum, and S. meliloti (Supplementary Table S3 in the Supplementary Materials).

\section{FnrN/FixK}

Transcription factors related to FnrN and FixK that could also be important for nitrogen fixation are distributed more widely than genes for the nitrogenase itself (Supplementary Table S3 in the Supplementary Materials). Some analyzed genomes contain multiple copies of $f n r N$ and/or fixK as usually more than one CrpFnr superfamily protein per genome could be found (Vollack et al., 1999; Korner et al., 2003;Matsui et al., 2013)

FixK and FnrN are very closely related, so we performed a detailed analysis of their orthologs, in order to be able to describe the evolution of regulatory cascades involving these factors. The FnrN/FixK orthologs were identified using the following procedure: (1) homologs of previously known FnrN/FixK were found in the analyzed genomes; (2) a phylogenetic tree for all found homologs was constructed; (3) only proteins forming monophyletic branches with known FnrN/FixK proteins were retained for further analysis (Figure 1; Supplementary Figure S1 in the Supplementary Materials).

As the presence of cysteine residues required for formation of oxygen-sensitive iron-sulfur clusters is a common feature of the Fnr branch of the Crp-Fnr superfamily (Korner et al., 2003; Matsui et al., 2013), to distinguish between FnrN-like and FixK-like regulators, we analyzed the detected proteins for the presence of such cysteine residues. The proteins retaining these cysteine residues formed a single monophyletic branch on the tree (Figure 1) and, hence, were annotated as FnrN orthologs. In addition to previously known FnrN proteins, this branch also contains the AadR protein (locus tag: RPA4234) from Rhodopseudomonas palustris (Dispensa et al., 1992; Egland and Harwood, 1999; Rey and Harwood, 2010), a transcription regulator of anaerobic degradation of aromatic acids (see below).

Proteins lacking the cysteine residues form two branches. One branch contains previously studied FixK proteins from various Rhizobiales, whereas the second branch includes the FixKf (locus tag: RHE_PF00508) protein of $R$. etli and is related to the FnrN branch (Figure 1). Because of that, the latter group cannot be considered as orthologs of FixK. On the other hand, the absence of cysteine residues required for formation of the ironsulfur cluster (Supplementary Figure S1 in the Supplementary Materials) shows that these proteins are not orthologs of FnrN. As FnrN and FixKf branches are clearly separated from each other, and both types of proteins are present in Rhizobium spp., we propose that FnrN and FixKf are divergent paralogs.

As the FixKf branch is much closer to FnrN than to FixK, we propose that FixKf and FixK independently have lost the ability to form the iron-sulfur cluster for performing the same functions. Thus, the FixKf proteins were defined as a separate group of transcription regulators. Hence, among FnrN/FixK-like proteins at least four groups of transcription regulators can be distinguished: (1) FnrN (also known as FnrL in R. sphaeroides) containing an iron-sulfur cluster, (2) regulator of anaerobic degradation of aromatic acids AadR, also containing an ironsulfur cluster, (3) FixK proteins lacking an iron-sulfur cluster, and (4) FixKf also lacking an iron-sulfur cluster, but much more closely related to FnrN than to FixK.

Overall, FnrN/FixK-like proteins were found in 43 of 50 analyzed genomes. The distribution of these proteins is taxonspecific. Caulobacteriales have only FixK proteins, whereas Rhodobacterales, Rhodospirillales, and Sphingomonadales have only FnrN proteins. In Rhizobiales, various combinations of FixK, FnrN, and FixKf were observed - only FixK in three genomes; only FnrN in two genomes; both FixK and FnrN in six genomes; FixKf and FnrN in two genomes; and FixK and FixKf in one genome (Supplementary Table S3 in the Supplementary Materials). In all studied Alphaproteobacteria, except Z. mobilis, FnrN/FixK-like proteins were found in all organisms having the nitrogenase genes.

\section{FixK/FixKf and FixLJ Co-occurrence}

As the FixK proteins lack the iron-sulfur cluster, they are activated in response to oxygen through the FixLJ-FixK regulatory cascade (Nellen-Anthamatten et al., 1998; Mesa et al., 2008; Reutimann et al., 2010). The fixK genes are present in all genomes having the fixLJ operon, the only exception is Rhizobium sp. NGR234, lacking the fixLJ operon. For the fixKf genes such correlation was not observed, as only in S. meliloti both fixKf and fixLJ genes were found (Supplementary Table S3 in the Supplementary Materials). 


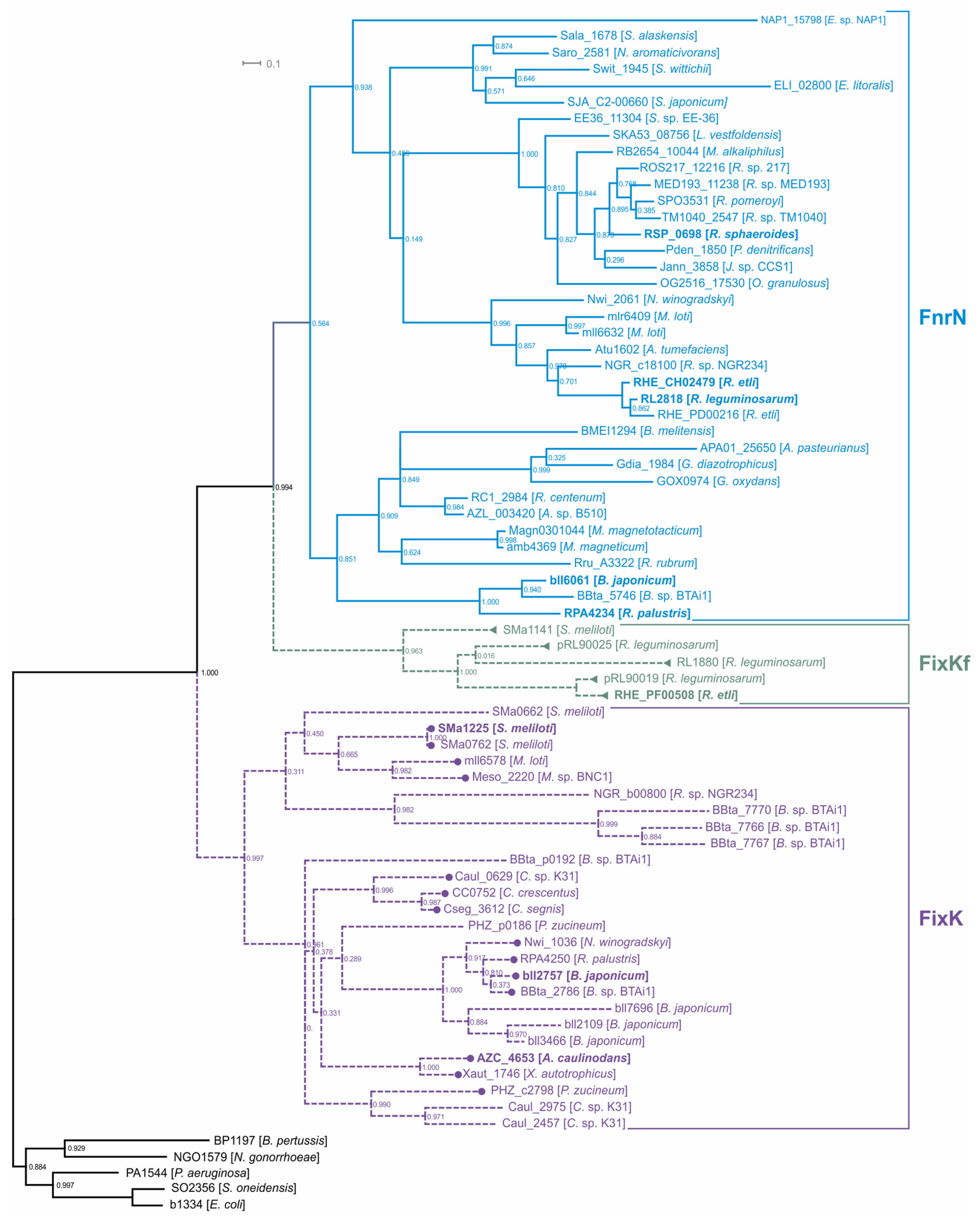

FIGURE 1 | Maximum-likelihood phylogenetic tree for FnrN/FixK-like proteins. Locus tags for proteins are shown. Previously analyzed proteins are shown in bold. Solid branches correspond to proteins that have cysteine residues for the iron-sulfur cluster, dashed branches correspond to proteins lacking such cysteine residues. Regulation by FixJ is shown by circles, regulation by FxkR, by triangles. The following proteins were used as the outgroups: the Fnr proteins from Bordetella pertussis Tohama I (BP1197), Escherichia coli K-12 MG1655 (b1334), Neisseria gonorrhoeae FA 1090 (NGO1579), and Shewanella oneidensis MR-1 (SO2356), and the Anr protein from Pseudomonas aeruginosa PAO1 (PA1544). 
A

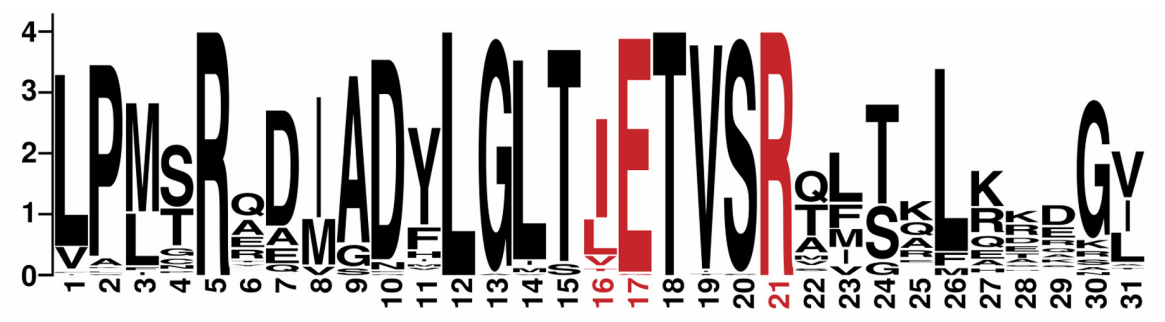

B
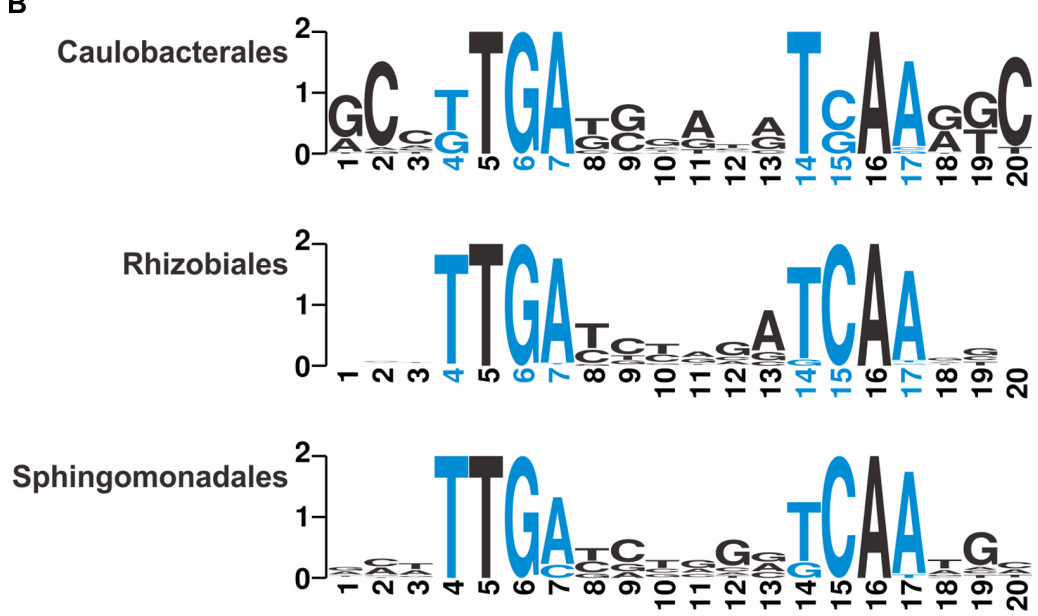

FixK
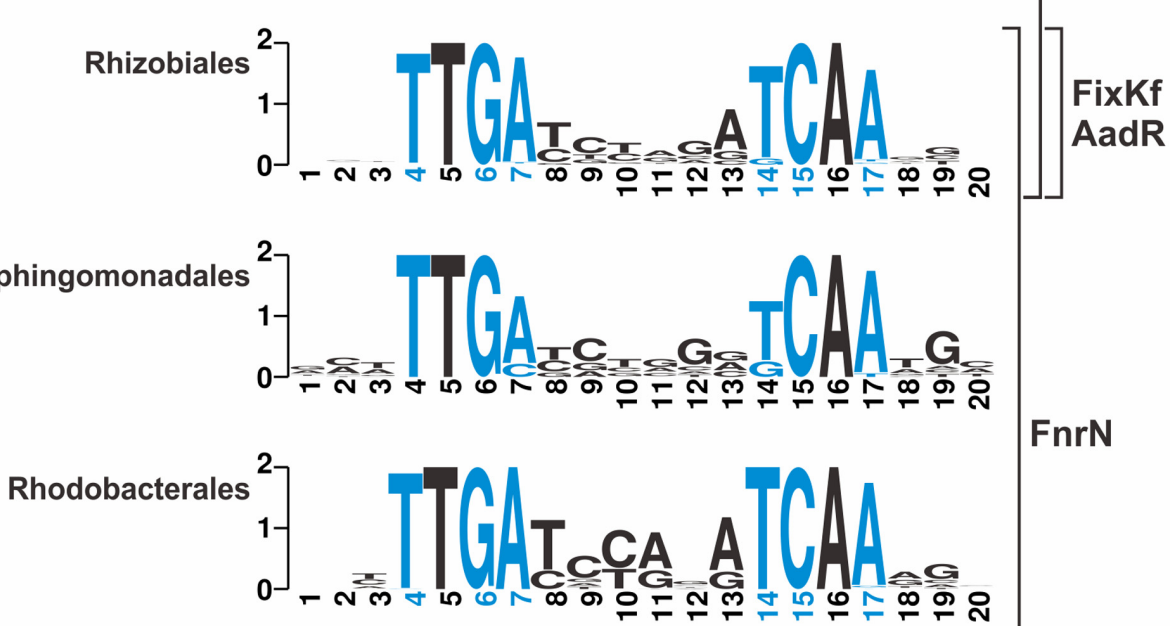

FnrN

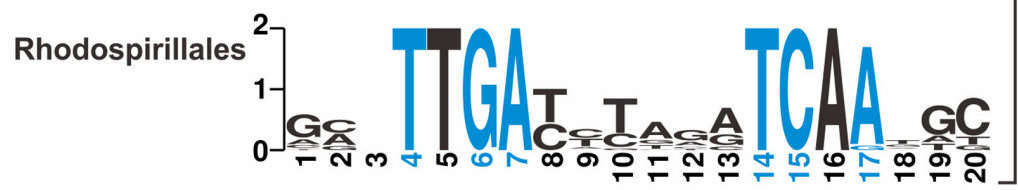

FIGURE 2 | Sequence Logos for (A) HTH domain of FixK/FnrN-like proteins and (B) their binding motifs. Positions for amino acids and nucleotides specifically interacting with each other are shown in red and blue, respectively. (A) The logo was constructed based on 65 proteins from 43 genomes.

(B) Taxon-specific logos were constructed for the following numbers of sites and genomes, Caulobacterales, 25 sites, 4 genomes; Rhizobiales, 192 sites, 14 genomes, Sphingomonadales, 23 sites, 5 genomes; Rhodobacterales, 85 sites, 12 genomes; Rhodospirillales, 18 sites, 8 genomes.

\section{Binding Motifs for Nitrogen-Fixation Transcription Regulators \\ NifA-Binding Motifs}

The initial profile for NifA-binding sites was constructed using previously known sites in R. etli (Salazar et al., 2010) and B. japonicum (Caldelari Baumberger et al., 2003; Hauser et al., 2007). Specific profiles were constructed for all four orders having nifA genes. Due to the degeneracy of the NifA-binding motif (Bueno et al., 2010; Salazar et al., 2010), the obtained profiles yielded a large number of false-positives. On the other hand, since NifA acts as a transcription activator only with the RpoN sigma-factor, we used prediction of RpoN promoters to eliminate false-positive NifA-binding sites. Thus, we constructed a profile using experimentally known RpoN promoters (Caldelari Baumberger et al., 2003; Hauser et al., 2007; Salazar et al., 2010). A predicted NifA-binding site was considered as a true positive only if it had a downstream predicted RpoNbinding site (Supplementary Table S4 in the Supplementary Materials).

\section{FnrN/FixK-Binding Motifs}

In an attempt to distinguish binding sites for FnrN/FixKlike regulators, we analyzed in detail sequences of these proteins and their binding sites (Figure 2). Amino acids involved in specific interactions with nucleotides of DNA sites (Bonnet et al., 2013) are highly conserved and no group-specific changes were found (Figure 2A; Supplementary 
A

Rhizobiales

AzC 4653

Xaut 1746

PHz cे2798

NWI 1036

RPA 4250

b112757

BBta_2786

Caulobacterales

Caul_0629

Cseg 3612

Cट्C 0752

B
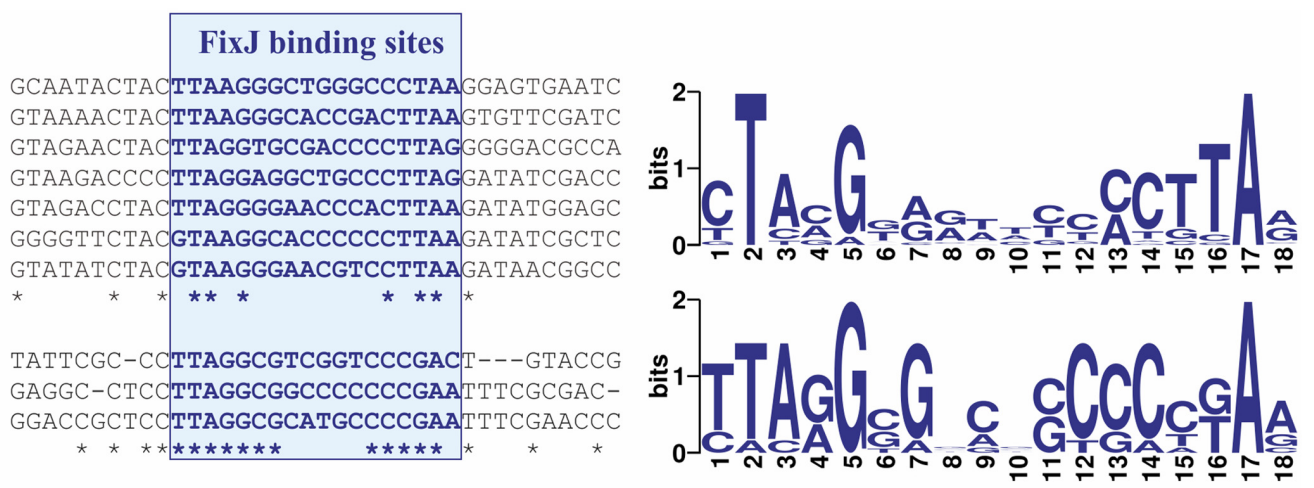

Rhizobiales

SMa1141 RHE_PF00508

pRL90019

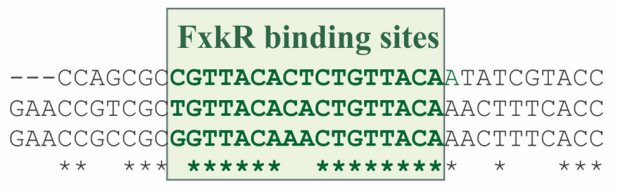

FIGURE 3 | Predicted binding sites for (A) FixJ and (B) FxkR proteins. Fragments of alignments for (A) fixK (10 genomes) and (B) fixKf (3 genomes) upstream regions (left) and sequence logos for binding motifs (right) are shown.

Figure S1 in the Supplementary Materials). Thus, we constructed taxon-specific profiles for FixK in Caulobacteriales and FnrN in Rhodobacterales, Rhodospirillales, and Sphingomonadales (Figure 2B). For Rhizobiales, we constructed one general profile to search for binding sites of all four regulators.

\section{FixJ-Binding Motif}

Since the fixK gene is the main target of FixJ-dependent regulation, to determine a profile for FixJ-binding sites, we analyzed upstream regions of the fixK genes. Phylogenetic footprinting revealed similar 18-bp imperfect palindromes in promoter regions from both Rhizobiales that coincides with the previously experimentally determined 16-bp sequence (Kurashima-Ito et al., 2005) and Caulobacteriales (Figure 3; Supplementary Figure S2 in the Supplementary Materials). Order-specific profiles were constructed for FixJ-binding motifs in Rhizobiales and Caulobacteriales.

\section{FxkR-Binding Motif}

To build a profile for the FxkR-binding motif, we applied the phylogenetic footprinting approach to fix $K f$ upstream regions. We identified a 17-bp palindromic motif that is similar to the 16bp motif determined previously (Figure 3; Supplementary Figure S2 in the Supplementary Materials) (Zamorano-Sanchez et al., 2012). Site search in FxkR-containing genomes revealed putative FxkR-binding sites upstream of not only fix $K f$, but also several other relevant genes (see below).

\section{Structure of the Analyzed Regulons: New Members}

The regulatory network for nitrogen fixation was reconstructed in 44 Alphaproteobacteria genomes having at least one of the analyzed regulators.

\section{NifA Regulon}

Genes encoding the NifA protein were found in 18 of 50 analyzed genomes. In each of these genomes NifA-binding sites were found upstream of multiple operons. In total, we found candidate NifA-binding sites upstream of 95 operons comprised of 280 genes. These genes include previously known targets such as the nif, fix, hup operons, groESL, iscN, fdxN, and rpoN (Supplementary Table $\mathrm{S} 1$ in the Supplementary Materials). In Rhodospirillales, we observed candidate NifAbinding sites upstream of 34 operons comprised of 165 genes. In Rhodobacterales and Sphingomonadales, we studied single nitrogen-fixing representatives, $R$. sphaeroides and $Z$. mobilis, respectively. $R$. sphaeroides has NifA-binding sites upstream of 26 genes from 7 operons, in Z. mobilis we predicted 24 genes from 4 operons to be under control of NifA. NifA regulation outside Rhizobiales has been experimentally studied only in A. brasilense, where it controls the expression of the nifE gene (Potrich et al., 2001). However, most targets predicted to be under the NifA control in Rhodospirillales, Rhodobacterales, and Sphingomonadales (such as nif, fix, ics $N$, and $f d x N$ genes) have been experimentally shown to be under the NifA regulation in Rhizobiales.

Several new NifA regulon members were identified. The first group of genes is involved in the adaptation to microoxic conditions. It includes genes encoding cytochrome P450 $(c p x)$, oxidoreductase $(f i x)$, that are under NifA regulation in several Rhizobiales; and alkyl hydroperoxide reductases

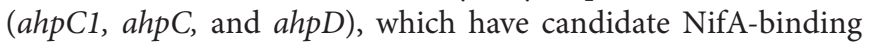
sites in some Rhizobiales and $R$. rubrum ATCC 11170. The second group includes the molybdenum transport genes $\bmod A B C D$. NifA-dependent regulation of these genes is important because the molybdenum cofactor is essential for functioning of the nitrogenase protein complex. The modABC 
operon is under NifA regulation in Bradyrhizobium spp., Rhizobium sp. NGR234, and Gluconacetobacter diazotrophicus, whereas modD has NifA-binding sites in $G$ diazotrophicus, $R$. sphaeroides, and Z. mobilis. The third heterogeneous group includes genes directly or indirectly involved in electron transfer. In Rhizobiales, ferredoxins $f d x B, f x r A$, fer 2 , Febinding protein hesB and Fe-responsive regulator irr have candidate NifA-binding sites. In Rhodospirillales, fer 2 also has a candidate NifA-binding site, and the electron transport complex rnfABCDGEH is also under NifA control. In Azospirillum sp. B510, ferredoxin-encoding $f d x B$ is located in a NifA-regulated operon. In Rhodobacterales and Sphingomonadales, the rnf operon also has candidate NifA-binding sites. The last group is represented in Magnetospirillum and Rhodospirillum, where candidate NifA-binding sites were found upstream of the draTG genes that are known to switch off the nitrogenase activity by covalently binding ADP-ribose (Moure et al., 2015).

We have observed conserved NifA-binding sites upstream of several genes whose connection to nitrogen fixation is not known. NifA-binding sites were found upstream of genes encoding stress-response transcription regulator (bolA), xanthine dehydrogenase $(x d h C)$, and outer membrane protein $(o m p W)$. At that, the latter was shown experimentally to be regulated by NifA in Mesorhizobium loti (Sullivan et al., 2013).

\section{FixJ Regulon}

Candidate FixJ-binding sites in Rhizobiales were found upstream of four genes, transcription regulators nifA and fixK, ferredoxinlike protein fixX, and L-proline 3-hydroxylase SMc03253. The regulation of all these genes except fixX has been previously shown experimentally (Supplementary Table S1 in the Supplementary Materials). A new target gene, fix $X$, is located in a regulated operon with nifA and is a part of the electron transfer fixABCX complex. In Caulobacteriales, conserved FixJ-binding sites were consistently found upstream of only one already known target, fixK.

\section{FxkR Regulon}

We also characterized the FxkR regulon in Rhizobiales. Its candidate binding sites were found upstream of 10 operons comprised of 21 genes including previously known target, $f i x K f$, and several new targets such as fxkR itself and the cco operon.

\section{FnrN/FixK Regulon}

As mentioned above, FnrN/FixK-like regulators have taxonspecific distribution. In Caulobacteriales, only FixK has been found and here it controls the expression of 70 genes from 21 operons. The function of these targets is limited to redox processes. They include previously known targets such as cytochrome oxidase $c c o$ and cyd operons, haem biosynthesis gene hemN, two-component system fixLJ, and the fixK gene itself. We also observed candidate FixK-binding sites upstream of new target genes involved in redox processes, cytochrome $c$ oxidase (ctaCDBXGE).
Only FnrN has been found in Rhodobacterales, Rhodospirillales, and Sphingomonadales. In Rhodobacterales, 171 genes from 71 operons are under the FnrN control, in Rhodospirillales, 21 genes from 17 operons, and in Sphingomonadales, 52 genes from 18 operons. Here the main function of the target genes is also limited to redox processes. Previously known targets include cytochrome oxidases (the cco operons), haem biosynthesis genes hemA and hemN, and the regulator $f n r N$ genes. Newly found targets are also involved in redox processes. In Magnetospirillum spp., FnrN controls expression of cytochrome $c 4$ precursor gene $c y c A$ and in several Rhodobacterales, cytochrome c551 peroxidase $c c p R$.

Rhizobiales employ the most complicated FnrN/FixKregulatory network and here the function of target genes is not limited to redox processes. Among genes involved in redox processes, FnrN/FixK-binding sites were found upstream of known targets such as the $c c o, c y d$ operons, haem formation genes hemA, hem $C$, and hemN, and some new ones, the cta operon, NADH-ubiquinone oxidoreductase (nuoABCDEFGHIJKLMN), decaheme cytochrome $c(m t r A)$, haem lyase operon $c c m I E F H$ responsible for haem incorporation into cytochrome oxidase. In R. palustris, FnrN/FixK-binding sites are located upstream of a gene for Fe-responsive regulator irr, implicating the wider role for these proteins in the regulation of redox processes.

Other FnrN/FixK targets play roles in various nitrogen metabolic processes other than nitrogen fixation as shown experimentally for B. japonicum (Supplementary Table S1 in the Supplementary Materials). We identified candidate FnrN/FixK-binding sites upstream of such genes not only in $B$. japonicum, but also in several other rhizobia genomes. Nitrate reductase (napEDABC) operon has FnrN/FixK-binding sites in Bradyrhizobium spp., Rhizobium sp. NGR234, and S. meliloti, genes for nitrous oxide reductase (nos), in Bradyrhizobium spp. and S. meliloti, and genes for nitrite reductase (nirKV), in nine Rhizobiales genomes.

In Rhizobiales, the FnrN/FixK-regulatory network becomes more subdivided as more genes for transcription factors turn out to be under their control. These transcription factors include not only FnrN/FixK themselves and FixLJ, but Crp-Fnr superfamily transcription factor AadR, NO respiration regulator NosR, and some uncharacterized transcription regulators from the Crp-Fnr superfamily.

We observed that FnrN/FixK might control the phbC gene encoding poly-beta-hydroxybutyrate (PBH) polymerase in some Rhizobiales. It was shown previously that phbC mutants of S. meliloti resulted in nodule formation delay in Medicago truncatula and young M. sativa nodules (Wang et al., 2007). PHB synthesis genes are downregulated in M. huakuii 7653R during symbiosis (Peng et al., 2014). PBH synthesis and accumulation was experimentally studied in nitrogen-fixing Gammaproteobacteria Azotobacter vinelandii and was shown to be involved in encystment (Stevenson and Socolofsky, 1966). A possible link between the PHB metabolism and nitrogen fixation has been suggested previously (Oelze, 2000), as in low oxygen concentration redundant carbon is stored as PHB rather than is dissimilated to provide reducing equivalents and energy for nitrogen fixation. 


\section{DISCUSSION}

\section{Varying Complexity of Nitrogen-Fixation Regulatory Cascades}

The analyzed genomes of Alphaproteobacteria demonstrate considerable variability in the repertoire of studied regulators and the composition of their regulons. Some nitrogen-fixing bacteria possess simple networks consisting of two independent regulons, nitrogen fixation genes under the NifA control and the genes involved in the adaptation to oxygen concentration under the FnrN control. These organisms include Rhodospirillales, R. sphaeroides, and Z. mobilis. Other Alphaproteobacteria have a larger repertoire of transcription factors and more complicated, interdependent cascades (Supplementary Figure S3 in the Supplementary Materials).

While members of Caulobacteriales do not fix nitrogen but contain orthologs of FixK/FnrN-like transcription factors regulating nitrogen fixation in other bacteria, indicating that the role of these regulatory systems is not so much in nitrogen fixation, as in adjustment of redox processes. All four Caulobacteriales species have the FixLJ system and FixK, and the following levels of the network complexity can be observed (Figure 4; Supplementary Figure S3 in the Supplementary Materials): (1) FixLJ controlling the fixK gene as in all studied Caulobacteriales); (2) additional autoregulation of fixK regulates its own gene as in Phenylobacterium zucineum, Caulobacter crescentus, and C. segnis); (3) further, FixK controlling fixJ, hence closing the feedback loop as in C. crescentus, C. segnis, and Caulobacter sp. K31).

The most complex regulatory network functions in Rhizobiales (Figure 4; Supplementary Figure S3 in the Supplementary Materials): (1) FixLJ is always on the top of the cascades and in all nitrogen-fixing bacteria it controls the fixK gene (Agron et al., 1993; Fischer, 1994; Robles et al., 2006). In the simplest case NifA controls nitrogen-fixation genes independently (B. japonicum, $B$. sp. BTAi, R. palustris). (2) In some bacteria (S. meliloti, M. loti, A. caulinodans, Xanthobacter autotrophicus) FixLJ controls the nifA and hence establishes a link between the nitrogen fixation process and oxygen concentration (Kaminski and Elmerich, 1991; Fischer, 1994). (3) An alternative two-component system, hFixL-FxkR may co-exist with FixLJ (S. meliloti) or substitute it (R. leguminosarum, R. etli). Analogously to FixLJ controlling fixK, this alternative hFixL-FxkR system controls the fixKf gene (Zamorano-Sanchez et al., 2012). (4) In the organisms

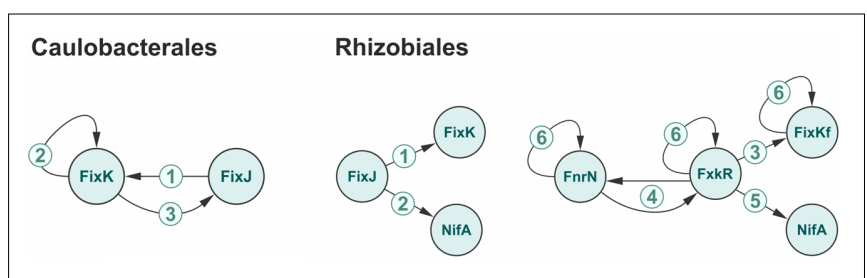

FIGURE 4 | Gradual growth of complexity for the nitrogen regulatory network. The numbers represent the levels of the network complexity. For the details see the text. with the alternative hFixL-FxkR two-component system (R. etli and $R$. leguminosarum), this system forms a loop with FnrN (Boesten and Priefer, 2004). (5) In R. leguminosarum, hFixLFxkR controls the nifA gene, $c f$. case (2), (6) Additionally, in some Rhizobiales several transcription factors regulate their own genes (Batut et al., 1989; Kaminski and Elmerich, 1998; NellenAnthamatten et al., 1998; Girard et al., 2000; Lopez et al., 2001; Martinez et al., 2004; Nukui et al., 2006; Dufour et al., 2010).

The comparative-genomics based approach used here allowed us to identify some novel transcriptional regulators in analyzed genomes, namely RPA1090 (Crp-Fnr family), CydR (ArsR family), and Bll2758 (OmpR family), based on their colocalization with nitrogen-fixation genes and/or regulation by nitrogen-fixation factors. However, each of these regulators was found in a small number of closely related genomes, precluding further comparative-genomic analysis of the corresponding regulons. However, we can distinguish between functionally significant conservancy, i.e., conservancy of gene context and/or predicted binding sites in a variety of genomes, from the overall conservancy caused by mere taxonomic proximity of the analyzed genomes. We expect that more sequenced genomes will make it possible to characterize binding motifs and cognate regulons for these regulators. Anyway, the narrow distribution of these regulators seems to point to their minor roles in the regulatory networks. Thus, we believe that the main observations made here are sufficiently robust.

\section{Evolution of Nitrogen Fixation-Related Regulatory Network: Major Events}

Based on our results and the species tree for the analyzed genomes, we attempted to reconstruct the evolutionary history of the analyzed regulons (Figure 5). Here we used the species tree extracted from the MicrobesOnline database. The species tree is based on concatenated protein sequences for 31 proteins universally present as single copies in complete bacterial genomes (Wu and Eisen, 2008). The evolutionary history of the analyzed regulons features numerous gains, losses, and horizontal gene transfers.

Almost all analyzed taxa (except Caulobacteriales) contain representatives that possess orthologs of $\mathrm{FnrN}$, so we propose that FnrN has been present in the last common ancestor of Alphaproteobacteria. Subsequently, it was lost in Caulobacteriales and substituted by the oxygen-sensing FixLJ system and the FixK regulator (Figure 5). The phylogenetic analysis of Caulobacteriales proteins for the FixLJ-FixK regulatory cascade placed these proteins within the Rhizobiales branch (Figure 1; Supplementary Figure S4 in the Supplementary Materials). Thus, the loss of the fnrN gene in Caulobacteriales was accompanied by horizontal transfer of the fixLJK gene cluster from a Rhizobiales organism. Most probably, the transfer of the fixLJK cluster preceded the loss of FnrN, and the expansion of the FixK regulon was due to binding of transferred FixK to existing FnrN-binding sites. This explains the similarity of the FixK regulon in Caulobacteriales and the FnrN regulon in Rhodobacterales, which are closely related to Caulobacteriales. 


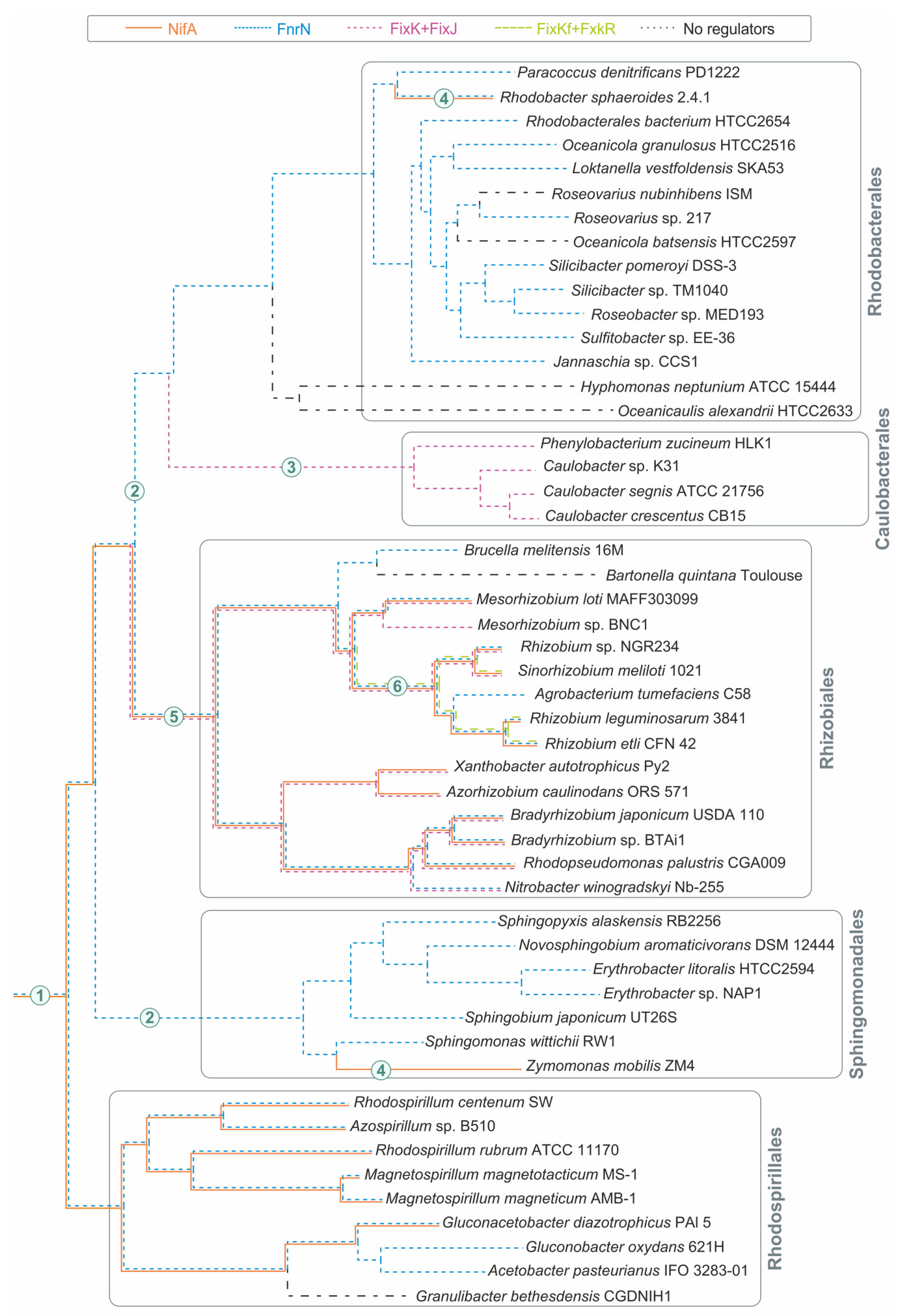

FIGURE 5 | Major evolutionary events in analyzed Alphaproteobacteria. The species tree was extracted from the MicrobesOnline web-resource (Dehal et al., 2010). The numbers on the branches represent major events: (1) NifA is a regulator of nitrogen fixation, FnrN is a regulator of aerobic respiration; (2) loss of NifA; (3) loss of FnrN, horizontal transfer of the fixLJ-fixK gene cluster from Rhizobiales, and expansion of the FixK regulon by the cyd and cco genes; (4) horizontal transfer of the nitrogen fixation locus from Rhodospirillales; (5) appearance of FixK and FixJ; (6) appearance of FixKf and FxkR. 
NifA orthologs are widespread in two distant branches (Rhizobiales and Rhodospirillales) and, hence also might be present in the common Alphaproteobacterial ancestor. Sphingomonadales and Rhodobacterales have single known members with NifA orthologs suggestive of horizontal gene transfer. The phylogenetic analysis of the NifA proteins (Supplementary Figure S5 in the Supplementary Materials) shows that NifA of Z. mobilis does not form a separate taxonspecific branch but is located within Rhodospirillales, clustering with the NifA protein from G. diazotrophicus Pal5. All NifAregulated genes in $\mathrm{Z}$. mobilis genome form a single chromosomal cluster (ZMO1808-37). This cluster includes the nifA gene itself, nitrogenase operon nifHDKENX-fdxB-nifQ, operons nifB-fdxN and iscN-nifUSVW-modD for nitrogenase maturation, and operon $r n f A B C D G E H$ for the electron transport complex. In G. diazotrophicus, NifA-regulated genes form an almost identical gene cluster, the only difference being the absence of the $r n f$ operon in G. diazotrophicus and the absence of the fixBAC genes in Z. mobilis. The phylogenetic analysis of NifA-regulated genes (only proteins of at least 200 amino acids were analyzed) demonstrated that the Nif and ModD proteins of Z. mobilis are clustered on the trees with the corresponding proteins from Rhodospirillales (Supplementary Figures S5B-J, in the Supplementary Materials), as well as modD and nif genes tends to form a single chromosomal cluster in analyzed Rhodospirillales genomes. Unfortunately, the $r n f$ operon is present in only four analyzed genomes and its phylogenetical analysis is irrelevant. Thus, based on the phylogeny of NifA, we propose that Z. mobilis obtained NifA together with operons nifHDKENX-fdxB-nifQ, $n i f B-f d x N$, and $i s c N-n i f U S V W-\bmod D$ via horizontal transfer from a Rhodospirillales organism. Regarding the $r n f$ operon, two alternative scenarios are possible, horizontal transfer together with NifA and other regulated genes, and expansion of the NifA regulon to the $r n f$ operon in $Z$. mobilis after horizontal transfer.

Rhodobacterales, $R$. sphaeroides might have gained nif gene cluster in a similar manner. In the phylogenetic tree, NifA from $R$. sphaeroides belongs to the Rhodospirillales branch clustering with NifA from Rhodospirillum centenum SW (Supplementary Figure S5A in the Supplementary Materials). In R. sphaeroides, the nifA gene is co-localized with NifA-regulated nif genes for nitrogen fixation (RSP_0532-46), whereas three other NifAregulated operons, rnf (RSP_3192-98), modD (RSP_2501), and RSP_2791-90, are located separately. In Rhodospirillales, the nif, $r n f$, and modD genes, but not RSP_2791-90, are also members of the NifA regulon. Phylogenetic analysis clusters most Nif proteins and ModD from $R$. sphaeroides with the proteins from Rhodospirillales (Supplementary Figures S5B-J, in the Supplementary Materials). Thus, we propose that the nif-modD cluster was transferred to R. sphaeroides from a Rhodospirillales genome, then the modD gene was re-located, and the NifA regulon expanded by the RSP_2791-90 operon. As for the rnf operon, both scenarios, horizontal transfer and regulon expansion, are possible, as in Z. mobilis (see above).

The evolutionary history of Rhizobiales is the most complicated among the studied taxa (Figure 5). The main event here might be recruitment to the network of the FixK protein and the FixLJ system in the common ancestor of Rhizobiales.
FixK appears to be the result of duplication of the ancestral $f n r N$ gene with subsequent loss of the iron-sulfur cluster. FixLJ might result from recruitment of an existing two-component system. An additional regulatory cascade, hFixL-FxkR-FixKf, appeared in the common ancestor of Rhizobiaceae, as seen now in Agrobacterium tumefaciens, Rhizobium spp., and S. meliloti. FixKf appears to be a paralog of $f n r N$ resulting from duplication in the common ancestor of Rhizobiaceae while the subsequent hFixL-FxkR system was recruited later.

\section{Why So Redundant?}

The response to molecular oxygen in Alphaproteobacteria is characterized by a seeming redundancy of regulators. Firstly, two different systems are used for oxygen sensing, FnrN, which senses oxygen directly via the iron-sulfur cluster, and regulatory cascades, FixLJ-FixK or hFixL-FxkR-FixKf. Secondly, in eight studied genomes these systems are present in multiple copies. For example, five copies of fixK gene were found in Bradyrhizobium sp. BTAil (Supplementary Table S3 in the Supplementary Materials). Based on the literature analysis (for references see below) and results of this study we suggest the following explanation for this redundancy.

The need for two systems, FnrN and FixLJ-FixK, may be explained by different subcellular localization of oxygen-sensing structures in these systems. At that, FnrN senses intracellular oxygen, whereas FixL/hFixL senses extracellular oxygen. Absence of extracellular oxygen indicates that bacterium is inside a nodule and the transcriptional profile should be adapted to this new environment. Additionally, regulation via a cascade yields large response time in comparison to regulation by a single one-component regulator. Firstly, activation by twocomponent regulatory system takes at least $75 \mathrm{~s}$ by itself (Gao and Stock, 2015). Secondly, in the case of regulatory cascade, this activation should be followed by transcription and translation of activated gene. The speed of RNA elongation during transcription in Bacteria is of about 40-80 nucleotides per second (Bremer and Yuan, 1968; Vogel and Jensen, 1994), whereas a maximal speed of translational elongation is 20 amino acids per second (Dennis and Bremer, 1974; Dennis and Nomura, 1974; Young and Bremer, 1976). The length of the shortest FixK protein analyzed in this work, BBta_7767 from Bradirhizobium sp. BTAil, is 153 amino acids. Thus, even if translation is coupled with transcription and both these processes are performed at a maximal speed, biosynthesis of even the shortest regulatory protein takes between 7 and 8 s. Taking into account that all other FixK proteins are longer, initiation of transcription and translation also should take time, and that transcription and translation coupling is relatively rare (Bakshi et al., 2012), we can be sure that real time of the response through this regulatory cascade is longer than the response resulting from regulation by a onecomponent regulator. Hence, FnrN is used for fast response to the concentration of intracellular oxygen, whereas FixLJ-FixK cascades are used for slower and long-term response to the concentration of extracellular oxygen. Additionally, at least in some Rhizobiales, the FixLJ two-component systems provide a link between oxygen sensing and nitrogen fixation. Thus, the nifA 
gene is regulated by FixJ in S. meliloti, M. loti, and A. caulinodans, and by FxkR in R. leguminosarum. Thus, the presence of two alternative systems, the FnrN and FixLJ-FixK cascades, is not a redundancy, but is necessary for fine-tuning of the oxygen response in changing environment.

The presence of multiple copies of regulators can be explained by two different, but not mutually exclusive hypotheses. The first one is that the presence of multiple copies provides protection against the regulator destruction. As the oxygen response plays a crucial role in nitrogen fixation, destruction of an oxygen-responsive transcription regulator may be extremely harmful. So, multiple oxygen-responsive regulators serve as backups. The second hypothesis is that different paralogs have different affinities to cognate binding sites in a genome. Binding of paralogous transcription regulators to the same binding sites with different affinities has been previously demonstrated for pairs of regulators from Escherichia coli, such as GalS/GalR (Geanacopoulos and Adhya, 1997), ExuR/UxuR (Suvorova et al., 2011), and NarL/NarP (Darwin et al., 1997). Similarly, copies of FnrN proteins may have different sensitivity to oxygen. Hence, they can differently change transcription levels of the regulated genes in response to varying levels of oxygen. Under this explanation, multiple copies of

\section{REFERENCES}

Agron, P. G., Ditta, G. S., and Helinski, D. R. (1993). Oxygen regulation of nifA transcription in vitro. Proc. Natl. Acad. Sci. U.S.A. 90, 3506-3510. doi: 10.1073/ pnas.90.8.3506

Altschul, S. F., Madden, T. L., Schaffer, A. A., Zhang, J., Zhang, Z., Miller, W., et al. (1997). Gapped BLAST and PSI-BLAST: a new generation of protein database search programs. Nucleic Acids Res. 25, 3389-3402. doi: 10.1093/nar/25.17.3389

Arcondeguy, T., Huez, I., Tillard, P., Gangneux, C., De Billy, F., Gojon, A., et al. (1997). The Rhizobium meliloti PII protein, which controls bacterial nitrogen metabolism, affects alfalfa nodule development. Genes Dev. 11, 1194-1206. doi: 10.1101/gad.11.9.1194

Bakshi, S., Siryaporn, A., Goulian, M., and Weisshaar, J. C. (2012). Superresolution imaging of ribosomes and RNA polymerase in live Escherichia coli cells. Mol. Microbiol. 85, 21-38. doi: 10.1111/j.1365-2958.2012.08081.x

Barrios, H., Valderrama, B., and Morett, E. (1999). Compilation and analysis of sigma(54)-dependent promoter sequences. Nucleic Acids Res. 27, 4305-4513. doi: 10.1093/nar/27.22.4305

Batut, J., Daveran-Mingot, M. L., David, M., Jacobs, J., Garnerone, A. M., and Kahn, D. (1989). fixK, a gene homologous with fnr and crp from Escherichia coli, regulates nitrogen fixation genes both positively and negatively in Rhizobium meliloti. EMBO J. 8, 1279-1286.

Boesten, B., and Priefer, U. B. (2004). The C-terminal receiver domain of the Rhizobium leguminosarum bv. viciae FixL protein is required for free-living microaerobic induction of the fnrN promoter. Microbiology 150, 3703-3713. doi: 10.1099/mic.0.27323-0

Bonnet, M., Kurz, M., Mesa, S., Briand, C., Hennecke, H., and Grutter, M. G. (2013). The structure of Bradyrhizobium japonicum transcription factor FixK2 unveils sites of DNA binding and oxidation. J. Biol. Chem. 288, 14238-14246. doi: 10.1074/jbc.M113.465484

Boyd, E. S., Costas, A. M., Hamilton, T. L., Mus, F., and Peters, J. W. (2015). Evolution of molybdenum nitrogenase during the transition from anaerobic to aerobic metabolism. J. Bacteriol. 197, 1690-1699. doi: 10.1128/JB.02611-14

Boyd, E. S., and Peters, J. W. (2013). New insights into the evolutionary history of biological nitrogen fixation. Front. Microbiol. 4:201. doi: 10.3389/ fmicb.2013.00201

Bremer, H., and Yuan, D. (1968). RNA chain growth-rate in Escherichia coli. J. Mol. Biol. 38, 163-180. doi: 10.1016/0022-2836(68)90404-X regulators form a mechanism for fine-tuning of the oxygen response.

\section{AUTHOR CONTRIBUTIONS}

DR and MG conceived and designed the research project. DR, OT, and JC performed the comparative genomic analysis to reconstruct regulons. DR, OT, and MG wrote the manuscript. All authors read and approved the final manuscript.

\section{ACKNOWLEDGMENT}

This study was supported by a grant from the Russian Science Foundation to MG (14-24-0155).

\section{SUPPLEMENTARY MATERIAL}

The Supplementary Material for this article can be found online at: http://journal.frontiersin.org/article/10.3389/fmicb. 2016.01343

Bueno, E., Mesa, S., Sanchez, C., Bedmar, E. J., and Delgado, M. J. (2010). NifA is required for maximal expression of denitrification genes in Bradyrhizobium japonicum. Environ. Microbiol. 12, 393-400. doi: 10.1111/j.1462-2920.2009.02076.x

Caldelari Baumberger, I., Fraefel, N., Gottfert, M., and Hennecke, H. (2003). New NodW- or NifA-regulated Bradyrhizobium japonicum genes. Mol. Plant Microbe Interact. 16, 342-351. doi: 10.1094/MPMI.2003.16.4.342

Cebolla, A., and Palomares, A. J. (1994). Genetic regulation of nitrogen fixation in Rhizobium meliloti. Microbiologia 10, 371-384.

Crooks, G. E., Hon, G., Chandonia, J. M., and Brenner, S. E. (2004). WebLogo: a sequence logo generator. Genome Res. 14, 1188-1190. doi: 10.1101/gr.849004

Darwin, A. J., Tyson, K. L., Busby, S. J., and Stewart, V. (1997). Differential regulation by the homologous response regulators NarL and NarP of Escherichia coli K-12 depends on DNA binding site arrangement. Mol. Microbiol. 25, 583-595. doi: 10.1046/j.1365-2958.1997.4971855.x

Dehal, P. S., Joachimiak, M. P., Price, M. N., Bates, J. T., Baumohl, J. K., Chivian, D., et al. (2010). MicrobesOnline: an integrated portal for comparative and functional genomics. Nucleic Acids Res. 38, D396-D400. doi: 10.1093/nar/gkp919

Dennis, P. P., and Bremer, H. (1974). Differential rate of ribosomal protein synthesis in Escherichia coli B/r. J. Mol. Biol. 84, 407-422. doi: 10.1016/00222836(74)90449-5

Dennis, P. P., and Nomura, M. (1974). Stringent control of ribosomal protein gene expression in Escherichia coli. Proc. Natl. Acad. Sci. U.S.A. 71, 3819-3823. doi: 10.1073/pnas.71.10.3819

Dispensa, M., Thomas, C. T., Kim, M. K., Perrotta, J. A., Gibson, J., and Harwood, C. S. (1992). Anaerobic growth of Rhodopseudomonas palustris on 4-hydroxybenzoate is dependent on AadR, a member of the cyclic AMP receptor protein family of transcriptional regulators. J. Bacteriol. 174, 5803-5813.

Disz, T., Akhter, S., Cuevas, D., Olson, R., Overbeek, R., Vonstein, V., et al. (2010). Accessing the SEED genome databases via Web services API: tools for programmers. BMC Bioinformatics 11:319. doi: 10.1186/1471-2105-11-319

Dixon, R., and Kahn, D. (2004). Genetic regulation of biological nitrogen fixation. Nat. Rev. Microbiol. 2, 621-632. doi: 10.1038/nrmicro954

Dos Santos, P. C., Fang, Z., Mason, S. W., Setubal, J. C., and Dixon, R. (2012). Distribution of nitrogen fixation and nitrogenase-like sequences amongst microbial genomes. BMC Genomics 13:162. doi: 10.1186/1471-2164-13-162 
Dufour, Y. S., Kiley, P. J., and Donohue, T. J. (2010). Reconstruction of the core and extended regulons of global transcription factors. PLoS Genetics 6:e1001027. doi: 10.1371/journal.pgen.1001027

Edgar, R. C. (2004). MUSCLE: multiple sequence alignment with high accuracy and high throughput. Nucleic Acids Res. 32, 1792-1797. doi: 10.1093/nar/gkh340

Egland, P. G., and Harwood, C. S. (1999). BadR, a new MarR family member, regulates anaerobic benzoate degradation by Rhodopseudomonas palustris in concert with AadR, an Fnr family member. J. Bacteriol. 181, 2102-2109.

Enkh-Amgalan, J., Kawasaki, H., and Seki, T. (2006). Molecular evolution of the nif gene cluster carrying nifI1 and nifI2 genes in the Gram-positive phototrophic bacterium Heliobacterium chlorum. Int. J. Syst. Evol. Microbiol. 56, 65-74. doi: 10.1099/ijs.0.63815-0

Felsenstein, J. (1996). Inferring phylogenies from protein sequences by parsimony, distance, and likelihood methods. Methods Enzymol. 266, 418-427. doi: 10.1016/S0076-6879(96)66026-1

Ferrieres, L., and Kahn, D. (2002). Two distinct classes of FixJ binding sites defined by in vitro selection. FEBS Lett. 517, 185-189. doi: 10.1016/S00145793(02)02618-2

Finn, R. D., Bateman, A., Clements, J., Coggill, P., Eberhardt, R. Y., Eddy, S. R., et al. (2014). Pfam: the protein families database. Nucleic Acids Res. 42, D222-D230. doi: $10.1093 /$ nar/gkt1223

Fischer, H. M. (1994). Genetic regulation of nitrogen fixation in rhizobia. Microbiol. Rev. 58, 352-386.

Galinier, A., Garnerone, A. M., Reyrat, J. M., Kahn, D., Batut, J., and Boistard, P. (1994). Phosphorylation of the Rhizobium meliloti FixJ protein induces its binding to a compound regulatory region at the fixK promoter. J. Biol. Chem. 268, 23784-23789.

Gallon, J. R. (1981). The oxygen sensitivity of nitrogenase: a problem for biochemists and micro-organisms. Trends Biochem. Sci. 6, 19-23. doi: 10.1016/0968-0004(81)90008-6

Gao, R., and Stock, A. M. (2015). Temporal hierarchy of gene expression mediated by transcription factor binding affinity and activation dynamics. MBio 6, e686-e615. doi: 10.1128/mBio.00686-15

Geanacopoulos, M., and Adhya, S. (1997). Functional characterization of roles of GalR and GalS as regulators of the gal regulon. J. Bacteriol. 179, 228-234.

Girard, L., Brom, S., Davalos, A., Lopez, O., Soberon, M., and Romero, D. (2000). Differential regulation of fixN-reiterated genes in Rhizobium etli by a novel fixL-fixK cascade. Mol. Plant Microbe Interact. 13, 1283-1292. doi: 10.1094/MPMI.2000.13.12.1283

Guindon, S., Dufayard, J. F., Lefort, V., Anisimova, M., Hordijk, W., and Gascuel, O. (2010). New algorithms and methods to estimate maximumlikelihood phylogenies: assessing the performance of PhyML 3.0. Syst. Biol. 59, 307-321. doi: 10.1093/sysbio/syq010

Hartmann, L. S., and Barnum, S. R. (2010). Inferring the evolutionary history of Mo-dependent nitrogen fixation from phylogenetic studies of nifK and nifDK. J. Mol. Evol. 71, 70-85. doi: 10.1007/s00239-010-9365-8

Hauser, F., Pessi, G., Friberg, M., Weber, C., Rusca, N., Lindemann, A., et al. (2007). Dissection of the Bradyrhizobium japonicum NifA+sigma54 regulon, and identification of a ferredoxin gene ( $\mathrm{fdxN})$ for symbiotic nitrogen fixation. Mol. Genet. Genomics 278, 255-271. doi: 10.1007/s00438-0070246-9

Hennecke, H. (1990). Nitrogen fixation genes involved in the Bradyrhizobium japonicum-soybean symbiosis. FEBS Lett. 268, 422-426. doi: 10.1016/00145793(90)81297-2

Huergo, L. F., Pedrosa, F. O., Muller-Santos, M., Chubatsu, L. S., Monteiro, R. A., Merrick, M., et al. (2012). PII signal transduction proteins: pivotal players in post-translational control of nitrogenase activity. Microbiology 158, 176-190. doi: 10.1099/mic.0.049783-0

Huson, D. H., Richter, D. C., Rausch, C., Dezulian, T., Franz, M., and Rupp, R. (2007). Dendroscope: an interactive viewer for large phylogenetic trees. BMC Bioinformatics 8:460. doi: 10.1186/1471-2105-8-460

Kaminski, P. A., and Elmerich, C. (1991). Involvement of fixLJ in the regulation of nitrogen fixation in Azorhizobium caulinodans. Mol. Microbiol. 5, 665-673. doi: 10.1111/j.1365-2958.1991.tb00820.x

Kaminski, P. A., and Elmerich, C. (1998). The control of Azorhizobium caulinodans nifA expression by oxygen, ammonia and by the HF-I-like protein. NrfA. Mol. Microbiol. 28, 603-613. doi: 10.1046/j.1365-2958.1998.00823.x
Kanehisa, M., Goto, S., Sato, Y., Kawashima, M., Furumichi, M., and Tanabe, M. (2014). Data, information, knowledge and principle: back to metabolism in KEGG. Nucleic Acids Res. 42, D199-D205. doi: 10.1093/nar/gkt1076

Korner, H., Sofia, H. J., and Zumft, W. G. (2003). Phylogeny of the bacterial superfamily of Crp-Fnr transcription regulators: exploiting the metabolic spectrum by controlling alternative gene programs. FEMS Microbiol. Rev. 27, 559-592. doi: 10.1016/S0168-6445(03)00066-4

Kurashima-Ito, K., Kasai, Y., Hosono, K., Tamura, K., Oue, S., Isogai, M., et al. (2005). Solution structure of the C-terminal transcriptional activator domain of FixJ from Sinorhizobium meliloti and its recognition of the fixK promoter. Biochemistry 44, 14835-14844. doi: 10.1021/bi0509043

Le, S. Q., and Gascuel, O. (2008). An improved general amino acid replacement matrix. Mol. Biol. Evol. 25, 1307-1320. doi: 10.1093/molbev/msn067

Lopez, O., Morera, C., Miranda-Rios, J., Girard, L., Romero, D., and Soberon, M. (2001). Regulation of gene expression in response to oxygen in Rhizobium etli: role of FnrN in fixNOQP expression and in symbiotic nitrogen fixation. J. Bacteriol. 183, 6999-7006. doi: 10.1128/JB.183.24.6999-7006.2001

Magrane, M., and Consortium, U. (2011). UniProt Knowledgebase: a hub of integrated protein data. Database (Oxford) 2011, bar009. doi: 10.1093/ database/bar009

Martinez, M., Palacios, J. M., Imperial, J., and Ruiz-Argueso, T. (2004). Symbiotic autoregulation of nifA expression in Rhizobium leguminosarum bv. viciae. J. Bacteriol. 186, 6586-6594. doi: 10.1128/JB.186.19.6586-6594.2004

Masepohl, B., Drepper, T., Paschen, A., Gross, S., Pawlowski, A., Raabe, K., et al. (2002). Regulation of nitrogen fixation in the phototrophic purple bacterium Rhodobacter capsulatus. J. Mol. Microbiol. Biotechnol. 4, 243-248.

Matsui, M., Tomita, M., and Kanai, A. (2013). Comprehensive computational analysis of bacterial CRP/FNR superfamily and its target motifs reveals stepwise evolution of transcriptional networks. Genome Biol. Evol. 5, 267-282. doi: 10.1093/gbe/evt004

McCue, L., Thompson, W., Carmack, C., Ryan, M. P., Liu, J. S., Derbyshire, V., et al. (2001). Phylogenetic footprinting of transcription factor binding sites in proteobacterial genomes. Nucleic Acids Res. 29, 774-782. doi: $10.1093 / \mathrm{nar} / 29.3 .774$

Mesa, S., Hauser, F., Friberg, M., Malaguti, E., Fischer, H. M., and Hennecke, H. (2008). Comprehensive assessment of the regulons controlled by the FixLJFixK2-FixK1 cascade in Bradyrhizobium japonicum. J. Bacteriol. 190, 65686579. doi: 10.1128/JB.00748-08

Mesa, S., Ucurum, Z., Hennecke, H., and Fischer, H. M. (2005). Transcription activation in vitro by the Bradyrhizobium japonicum regulatory protein FixK2. J. Bacteriol. 187, 3329-3338. doi: 10.1128/JB.187.10.3329-3338.2005

Mironov, A. A., Vinokurova, N. P., and Gel'fand, M. S. (2000). Software for analyzing bacterial genomes. Mol. Biol. (Mosk). 34, 253-262. doi: 10.1007/BF02759643

Moure, V. R., Costa, F. F., Cruz, L. M., Pedrosa, F. O., Souza, E. M., Li, X. D., et al. (2015). Regulation of nitrogenase by reversible mono-ADP-ribosylation. Curr. Top. Microbiol. Immunol. 384, 89-106. doi: 10.1007/82_2014_380

Nellen-Anthamatten, D., Rossi, P., Preisig, O., Kullik, I., Babst, M., Fischer, H. M., et al. (1998). Bradyrhizobium japonicum FixK2, a crucial distributor in the FixLJ-dependent regulatory cascade for control of genes inducible by low oxygen levels. J. Bacteriol. 180, 5251-5255.

Novichkov, P. S., Kazakov, A. E., Ravcheev, D. A., Leyn, S. A., Kovaleva, G. Y., Sutormin, R. A., et al. (2013). RegPrecise 3.0 - a resource for genome-scale exploration of transcriptional regulation in bacteria. BMC Genomics 14:745. doi: 10.1186/1471-2164-14-745

Novichkov, P. S., Rodionov, D. A., Stavrovskaya, E. D., Novichkova, E. S., Kazakov, A. E., Gelfand, M. S., et al. (2010). RegPredict: an integrated system for regulon inference in prokaryotes by comparative genomics approach. Nucleic Acids Res. 38, W299-W307. doi: 10.1093/nar/gkq531

Nukui, N., Minamisawa, K., Ayabe, S., and Aoki, T. (2006). Expression of the 1-aminocyclopropane-1-carboxylic acid deaminase gene requires symbiotic nitrogen-fixing regulator gene nifA2 in Mesorhizobium loti MAFF303099. Appl. Environ. Microbiol. 72, 4964-4969. doi: 10.1128/AEM.02745-05

Oelze, J. (2000). Respiratory protection of nitrogenase in Azotobacter species: is a widely held hypothesis unequivocally supported by experimental evidence? FEMS Microbiol. Rev. 24, 321-333. doi: 10.1111/j.1574-6976.2000.tb00545.x

Peng, J., Hao, B., Liu, L., Wang, S., Ma, B., Yang, Y., et al. (2014). RNA-Seq and microarrays analyses reveal global differential transcriptomes of Mesorhizobium 
huakuii 7653R between bacteroids and free-living cells. PLoS ONE 9:e93626. doi: 10.1371/journal.pone.0093626

Potrich, D. P., Bressel, T. A., Schrank, I. S., and Passaglia, L. M. (2001). Sequencing and promoter analysis of the nifENXorf3orf5fdxAnifQ operon from Azospirillum brasilense Sp7. Braz. J. Med. Biol. Res. 34, 1379-1395. doi: 10.1590/S0100-879X2001001100003

Ravcheev, D. A., Gerasimova, A. V., Mironov, A. A., and Gelfand, M. S. (2007). Comparative genomic analysis of regulation of anaerobic respiration in ten genomes from three families of gamma-proteobacteria (Enterobacteriaceae. Pasteurellaceae, Vibrionaceae). BMC Genomics 8:54. doi: 10.1186/1471-21648-54

Raymond, J., Siefert, J. L., Staples, C. R., and Blankenship, R. E. (2004). The natural history of nitrogen fixation. Mol. Biol. Evol. 21, 541-554. doi: 10.1093/molbev/msh047

Rehder, D. (2000). Vanadium nitrogenase. J. Inorg. Biochem. 80, 133-136. doi: 10.1016/S0162-0134(00)00049-0

Reutimann, L., Mesa, S., and Hennecke, H. (2010). Autoregulation of fixK(2) gene expression in Bradyrhizobium japonicum. Mol. Genet. Genomics 284, 25-32. doi: $10.1007 / \mathrm{s} 00438-010-0547-2$

Rey, F. E., and Harwood, C. S. (2010). FixK, a global regulator of microaerobic growth, controls photosynthesis in Rhodopseudomonas palustris. Mol. Microbiol. 75, 1007-1020. doi: 10.1111/j.1365-2958.2009.07037.x

Reyrat, J. M., David, M., Blonski, C., Boistard, P., and Batut, J. (1993). Oxygenregulated in vitro transcription of Rhizobium meliloti nifA and fixK genes. J. Bacteriol. 175, 6867-6872.

Robles, E. F., Sanchez, C., Bonnard, N., Delgado, M. J., and Bedmar, E. J. (2006). The Bradyrhizobium japonicum napEDABC genes are controlled by the FixLJ-FixK(2)-NnrR regulatory cascade. Biochem. Soc. Trans. 34, 108-110. doi: 10.1042/BST0340108

Rodgers, T. L., Townsend, P. D., Burnell, D., Jones, M. L., Richards, S. A., Mcleish, T. C., et al. (2013). Modulation of global low-frequency motions underlies allosteric regulation: demonstration in CRP/FNR family transcription factors. PLoS Biol. 11:e1001651. doi: 10.1371/journal.pbio.1001651

Rodionov, D. A. (2007). Comparative genomic reconstruction of transcriptional regulatory networks in bacteria. Chem. Rev. 107, 3467-3497. doi: 10.1021/ cr068309+

Salazar, E., Diaz-Mejia, J. J., Moreno-Hagelsieb, G., Martinez-Batallar, G., Mora, Y., Mora, J., et al. (2010). Characterization of the NifA-RpoN regulon in Rhizobium etli in free life and in symbiosis with Phaseolus vulgaris. Appl. Environ. Microbiol. 76, 4510-4520. doi: 10.1128/AEM.02007-09

Sciotti, M. A., Chanfon, A., Hennecke, H., and Fischer, H. M. (2003). Disparate oxygen responsiveness of two regulatory cascades that control expression of symbiotic genes in Bradyrhizobium japonicum. J. Bacteriol. 185, 5639-5642. doi: 10.1128/JB.185.18.5639-5642.2003

Seefeldt, L. C., Hoffman, B. M., and Dean, D. R. (2009). Mechanism of Mo-dependent nitrogenase. Annu. Rev. Biochem. 78, 701-722. doi: 10.1146/annurev.biochem.78.070907.103812

Shelton, D. A., Stegman, L., Hardison, R., Miller, W., Bock, J. H., Slightom, J. L., et al. (1997). Phylogenetic footprinting of hypersensitive site 3 of the beta-globin locus control region. Blood 89, 3457-3469.

Sousa, F. L., Parente, D. J., Shis, D. L., Hessman, J. A., Chazelle, A., Bennett, M. R., et al. (2015). AlloRep: a repository of sequence, structural and mutagenesis data for the LacI/GalR transcription regulators. J. Mol. Biol. 428, 671-678. doi: 10.1016/j.jmb.2015.09.015
Stevenson, L. H., and Socolofsky, M. D. (1966). Cyst formation and poly-betahydroxybutyric acid accumulation in Azotobacter. J. Bacteriol. 91, 304-310.

Sullivan, J. T., Brown, S. D., and Ronson, C. W. (2013). The NifA-RpoN regulon of Mesorhizobium loti strain R7A and its symbiotic activation by a novel LacI/GalR-family regulator. PLoS ONE 8:e53762. doi: 10.1371/ journal.pone.0053762

Sullivan, J. T., Trzebiatowski, J. R., Cruickshank, R. W., Gouzy, J., Brown, S. D., Elliot, R. M., et al. (2002). Comparative sequence analysis of the symbiosis island of Mesorhizobium loti strain R7A. J. Bacteriol. 184, 3086-3095. doi: 10.1128/JB.184.11.3086-3095.2002

Suvorova, I. A., Tutukina, M. N., Ravcheev, D. A., Rodionov, D. A., Ozoline, O. N., and Gelfand, M. S. (2011). Comparative genomic analysis of the hexuronate metabolism genes and their regulation in gammaproteobacteria. J. Bacteriol. 193, 3956-3963. doi: 10.1128/JB.00277-11

Torres, M. J., Bueno, E., Mesa, S., Bedmar, E. J., and Delgado, M. J. (2011). Emerging complexity in the denitrification regulatory network of Bradyrhizobium japonicum. Biochem. Soc. Trans. 39, 284-288. doi: 10.1042/BST0390284

Vogel, U., and Jensen, K. F. (1994). The RNA chain elongation rate in Escherichia coli depends on the growth rate. J. Bacteriol. 176, 2807-2813.

Vollack, K. U., Hartig, E., Korner, H., and Zumft, W. G. (1999). Multiple transcription factors of the FNR family in denitrifying Pseudomonas stutzeri: characterization of four fnr-like genes, regulatory responses and cognate metabolic processes. Mol. Microbiol. 31, 1681-1694. doi: 10.1046/j.13652958.1999.01302.x

Wang, C., Saldanha, M., Sheng, X., Shelswell, K. J., Walsh, K. T., Sobral, B. W., et al. (2007). Roles of poly-3-hydroxybutyrate (PHB) and glycogen in symbiosis of Sinorhizobium meliloti with Medicago sp. Microbiology 153, 388-398. doi: 10.1099/mic.0.29214-0

$\mathrm{Wu}, \mathrm{M}$, and Eisen, J. A. (2008). A simple, fast, and accurate method of phylogenomic inference. Genome Biol. 9, R151. doi: 10.1186/gb-2008-910 -r151

Young, R., and Bremer, H. (1976). Polypeptide-chain-elongation rate in Escherichia coli $\mathrm{B} / \mathrm{r}$ as a function of growth rate. Biochem. J. 160, 185-194. doi: 10.1042/bj1600185

Yurgel, S. N., Rice, J., Mulder, M., and Kahn, M. L. (2010). GlnB/GlnK PII proteins and regulation of the Sinorhizobium meliloti Rm1021 nitrogen stress response and symbiotic function. J. Bacteriol. 192, 2473-2481. doi: 10.1128/JB. 01657-09

Zamorano-Sanchez, D., Reyes-Gonzalez, A., Gomez-Hernandez, N., Rivera, P., Georgellis, D., and Girard, L. (2012). FxkR provides the missing link in the fixLfixK signal transduction cascade in Rhizobium etli CFN42. Mol. Plant Microbe Interact. 25, 1506-1517. doi: 10.1094/MPMI-05-12-0136-R

Conflict of Interest Statement: The authors declare that the research was conducted in the absence of any commercial or financial relationships that could be construed as a potential conflict of interest.

Copyright $\odot 2016$ Tsoy, Ravcheev, Čuklina and Gelfand. This is an open-access article distributed under the terms of the Creative Commons Attribution License (CC BY). The use, distribution or reproduction in other forums is permitted, provided the original author(s) or licensor are credited and that the original publication in this journal is cited, in accordance with accepted academic practice. No use, distribution or reproduction is permitted which does not comply with these terms. 